\section{Vigilância entomológica da doença de Chagas na região centro-oeste de Minas Gerais, Brasil, entre os anos de 2000 e 2003}

\author{
Entomological surveillance for Chagas disease \\ in the mid-western region of Minas Gerais State, \\ Brazil, from 2000 to 2003
}

\author{
1 Centro de Pesquisas René \\ Rachou, Fundação Oswaldo \\ Cruz, Belo Horizonte, Brasil. \\ 2 Diretoria de Ações \\ Descentralizadas \\ da Saúde de Divinópolis, \\ Divinópolis, Brasil. \\ Correspondência \\ M. M. Villela \\ Centro de Pesquisas René \\ Rachou, Fundação Oswaldo \\ Cruz. C. P. 1743, \\ Belo Horizonte, $M G$ \\ 30190-002, Brasil. \\ villela@cpqrr.fiocruz.br
}

\section{Abstract}

In Minas Gerais State, Brazil, after the elimination of Triatoma infestans by the Brazilian Chagas Disease Control Program, some triatomine species, such as Panstrongylus megistus, have merited the attention of entomological surveillance authorities. After the decentralization of the Brazilian health system, certain administrative and operational difficulties have been observed in some municipalities (counties). This study aims to evaluate entomological surveillance for Chagas disease in the 54 municipalities supervised by the Division of Decentralized Health Activities in Divinópolis, Minas Gerais, from 2000 to 2003. In 46 municipalities, 1,531 triatomines were captured, of which $94.1 \%$ were P. megistus. The infection rate for $\mathrm{T}$. cruzi in the vectors was $1.3 \%$. A total of 850 reports were received and 835 site visits were conducted, showing that the program is active and efficient in the majority of the municipalities analyzed. The importance of the maintenance and improvement of entomological surveillance in the region was demonstrated, since triatomine bugs continue to invade (and can colonize) the domiciliary environment, although with low density.

Chagas Disease; Epidemiologic Surveillance; Panstrongylus

\author{
Marcos M. Villela 1 \\ Janice B. Souza 2 \\ Vicente P. Mello 2 \\ Bernardino Vaz de Melo Azeredo ${ }^{2}$ \\ João Carlos P. Dias ${ }^{1}$
}

\section{Introdução}

A doença de Chagas é uma doença endêmica da América Latina, onde cerca de 90 milhões de pessoas vivem na área de risco de transmissão ${ }^{1}$. No Brasil, a doença acomete cerca de 3 milhões de pessoas 2 . Não havendo vacina que previna a infecção, a principal forma de evitar o aparecimento de novos casos reside no controle da transmissão vetorial, transfusional e congênita. Um dos desdobramentos importantes do controle vetorial é seu impacto positivo sobre a transmissão sangüínea e congênita, que, em última análise, são dela dependentes 3 .

No Brasil, a doença distribui-se em vários estados, sua ocorrência depende basicamente da distribuição dos vetores domiciliados 4,5,6. Minas Gerais foi um dos Estados brasileiros com maior prevalência da endemia chagásica, sendo, coincidentemente, onde a doença foi descoberta 7 . Nos anos 70, estimava-se a existência de 1 milhão de pessoas infectadas no Estado $8,9,10$.

Inicialmente, a espécie de triatomíneo que apresentava maior importância epidemiológica em Minas Gerais era Panstrongylus megistus, inseto nativo na área, onde pode ser encontrado invadindo casas e peridomicílio em grande dispersão. Já Triatoma infestans ingressou no Estado pela região sul e do Triângulo Mineiro, através de São Paulo, ao que tudo indica, no final do século XIX, chegando, em 1980, 
a representar mais de $70,0 \%$ da fauna domiciliar de triatomíneos em Minas Gerais 6,11,12.

A região oeste de Minas Gerais sempre apresentou significativa endemicidade para doença de Chagas humana. Historicamente, nessa área, destaca-se o Município de Bambuí, fonte de importantes estudos sobre a doença de Chagas humana e seu controle. Nessa cidade, não houve registro de T. infestans até 1939, tendo sido até então $P$. megistus o principal vetor de Trypanosoma cruzi. Entre 1940 e 1944, já se encontrava um total predomínio de T. infestans, sendo esse responsável por mais de $90,0 \%$ das infestações domiciliares encontradas 13,14,15. Sistemáticos estudos longitudinais mostraram que a espécie foi se dispersando até chegar à região norte do Estado, no sentido centro-sul, ocupando espaços onde somente $P$. megistus transmitia a tripanossomíase americana 12,16,17.

Entre 1975 e 1980, 440 municípios mineiros estavam infestados por triatomíneos, sendo que $40,0 \%$ desses possuíam T. infestans invadindo e colonizando vivendas humanas 10,12. As ações de controle da transmissão vetorial da doença de Chagas em Minas Gerais começaram, experimentalmente, a partir da década de 40, em Bambuí, objetivando diminuir a transmissão vetorial no âmbito domiciliar. O programa nacional foi institucionalizado, em 1950, pelo então Serviço Nacional de Malária e continuado pelo Departamento Nacional de Endemias Rurais, mas foi sistematizado e estruturado na forma de programa de alcance nacional, a partir de 1975, pela Superintendência de Campanhas de Saúde Pública (SUCAM), posteriormente, Fundação Nacional de Saúde (FUNASA). Até os anos 70, apenas o Estado de São Paulo mantinha ações regulares de controle, em paralelo com trabalhos pioneiros de investigação em Bambuí 13,18,19,20.

Baseada nos trabalhos clássicos da luta antimalárica e no modelo inicial desenvolvido em Bambuí, a estrutura básica do Programa de Controle da Doença de Chagas (PCDCh) formulouse, originalmente, em três fases: (a) planejamento e reconhecimento geográfico, (b) fase de ataque ao vetor domiciliado e (c) vigilância epidemiológica para evitar o retorno do vetor 21 . Outros fatores, além do uso de inseticidas, também colaboraram para que os resultados fossem favoráveis ao controle da endemia, como o êxodo rural ocorrido a partir da década de 50, devido à avalanche do modelo urbano-industrial, à onda da globalização e à economia de mercado, que inviabilizaram o arcaico sistema de produção rural, privilegiando agroindústrias, modernização, redução de mão-de-obra etc. 22 . Embora priorizado em 1983, o PCDCh teve suas atividades perturbadas, a partir de 1986, em virtude das repetidas epidemias de dengue no país e da perda de pessoal que foi remanejado para o controle dessa virose 23 .

Com a consolidação das ações da fase de ataque, a vigilância entomológica foi instituída em áreas de infestação residual, e a participação da população na detecção de triatomíneos em seus domicílios foi iniciada em todo o Brasil 24.

Um fato importante ocorreu com o PCDCh, em 1999, a partir da Portaria n. 1.399 do Ministério da Saúde, de 15 de dezembro de 1999 (Diário Oficial da União 1999; 16 dez.), que equacionou a descentralização das ações de controle de endemia e promoveu a transferência das responsabilidades para o Estado e municípios. Os programas de controle de endemias foram municipalizados e começaram a ser conduzidos pelas prefeituras municipais sob a coordenação das Diretorias de Ações Descentralizadas de Saúde (DADS). Em Minas Gerais, essas DADS pertencem à Secretaria Estadual de Saúde e estão sediadas em municípios de maior importância política ou econômica, existindo, hoje, 27 DADS. A elas compete a preparação de recursos humanos a partir da realização de cursos de capacitação dos agentes municipais, além da supervisão e normatização dos programas já implantados. Os recursos chegam às Diretorias Regionais a partir da FUNASA ou da Secretaria Nacional de Saúde e são repassados aos municípios mediante planejamento e negociação, numa Comissão Bipartite (composta entre Estado e municípios).

Objetivando contribuir para a análise e aprimoramento do PCDCh nesta nova realidade operacional e administrativa, o presente trabalho relata as ações de vigilância epidemiológica da doença de Chagas nos 54 municípios abrangidos pela DADS de Divinópolis - Minas Gerais, entre os anos de 2000 e 2003. Essa região foi escolhida por ser um dos mais importantes focos da tripanossomíase americana, alcançando elevados índices de prevalência da infecção humana no inquérito nacional de 19751980. Além do alto índice de infestação triatomínica dos domicílios, pois 51 dos 54 municípios possuíam habitações infestadas por $P$. megistus e outras espécies como: T. infestans, Triatoma pseudomaculata, Triatoma sordida, Rhodnius neglectus, Panstrongylus geniculatus e Panstrongylus diasi ${ }^{6}$. Além disso, essa DADS 
foi escolhida por estar bem estruturada e em pleno funcionamento, dispondo-se de bons dados históricos sobre a epidemiologia e controle da doença de Chagas.

\section{Materiais e métodos}

A área estudada corresponde a 54 municípios (Figura 1) da região centro-oeste de Minas Gerais, os quais são supervisionados pela DADS

\section{Figura 1}

Mapa dos 54 municípios que fazem parte da região centro-oeste de Minas Gerais, Brasil, coordenados pela DADS de Divinópolis, destacando-se a sede regional.
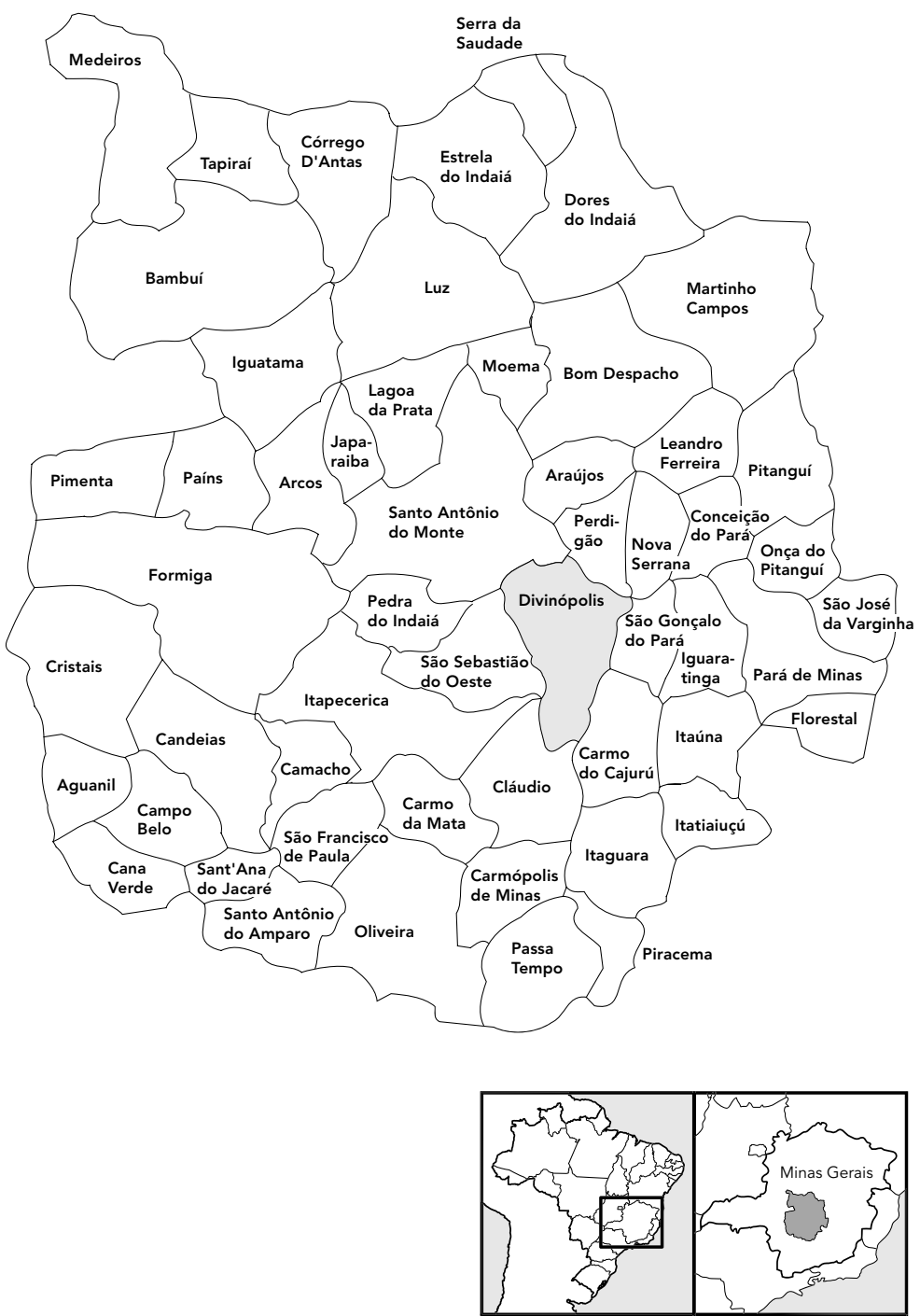

$1 \mathrm{~cm}=37 \mathrm{Km}$ de Divinópolis. A região apresenta 1.028.823 habitantes, sendo que $85,8 \%$ desses residem na zona urbana (Instituto Brasileiro de Geografia e Estatística. http://www.ibge.gov.br). Todos os municípios estão com PCDCh implantado e em fase de vigilância epidemiológica.

Os dados entomológicos utilizados nesta análise foram produzidos pela DADS de Divinópolis, a partir de relatórios confeccionados regularmente através de informes dos municípios e supervisões rotineiras dos inspetores regionais.

\section{Aspectos da vigilância}

Seguem-se basicamente as normativas clássicas da FUNASA 25. Os insetos são capturados através de um sistema de notificação de triatomíneos, realizado pela própria população, somada a busca ativa por agentes de saúde nas unidades domiciliares (UD) notificadas e seus arredores. Cada UD é constituída pela casa e seus anexos (todo o peridomicílio). Os moradores são instruídos a, quando encontrar insetos suspeitos em sua UD, capturá-los e enviálos para algum Posto de Informação de Triatomíneo (PIT), os quais estão instalados em locais estratégicos dos municípios, como: escolas, postos de saúde etc. Após a notificação, num prazo máximo de um mês, os agentes municipais capacitados visitam os domicílios e realizam pesquisa triatomínica durante o atendimento, com o auxílio de uma pinça e utilização de uma lanterna para melhor visualização dos esconderijos desses insetos. Os insetos também são capturados durante a realização da Pesquisa Integral (PI), na qual se realiza a captura de triatomíneos em todas as UDs existentes na zona rural do município analisado. Os triatomíneos encontrados no intra e no peridomicílio são colocados em recipientes diferentes e entregues para o laboratório da DADS sediado também em Divinópolis. Confirmada a notificação pelo agente nas UDs onde são encontrados triatomíneos durante o atendimento, faz-se a borrifação de toda a unidade com inseticidas piretróides.

No laboratório, os insetos recebidos foram classificados por espécie e estádio evolutivo, sendo que a captura de ninfas é interpretada como uma colonização no intra ou peridomicílio. Uma amostra dos insetos foi analisada para a presença de tripanossomos semelhantes ao T. cruzi, através do estudo microscópico do conteúdo intestinal dos insetos.

Com fins estatísticos, foram realizados testes $\chi^{2}$, considerando-se significantes os valores de $\mathrm{p}$ iguais ou menores que 0,05. 


\section{Resultados}

Através das notificações, atendimentos e PI, foram identificadas quatro espécies de triatomíneos: P. megistus, P. diasi, T. sordida e R. neglectus. No que diz respeito à colonização, P. megistus foi a espécie que apresentou maior índice, pois $32,3 \%$ dos exemplares capturados eram ninfas, sendo essa a única espécie a apresentar ninfas no intradomicílio. Em uma única ocasião, foram encontradas ninfas de T. sordida colonizando o peridomicílio, no Município de Arcos.

A espécie de maior importância epidemiológica, P. megistus, foi capturada em 43 dos 46 municípios que realizaram notificações, sendo eles: Aguanil, Bambuí, Bom Despacho, Camacho, Campo Belo, Cana Verde, Candeias, Carmo da Mata, Carmo do Cajuru, Carmópolis de Minas, Cláudio, Conceição do Pará, Córrego D’Antas, Córrego Fundo, Cristais, Dores do Indaiá, Formiga, Iguatama, Itaguara, Itapecirica, Itaúna, Itatiaiuçu, Leandro Ferreira, Luz, Martinho Campos, Medeiros, Nova Serrana, Oliveira, Onça do Pintangui, Paíns, Pará de Minas, Passa Tempo, Pedra do Indaiá, Perdigão, Pimenta, Piracema, Pitangui, Santo Antônio do Amparo, Santo Antônio do Monte, São Francisco de Paula, São Sebastião do Oeste, Serra da Saudade e Tapiraí. Em oito municípios, nenhuma notificação foi realizada, sendo esses: Araújos, Igaratinga, Japaraiba, Lagoa da Prata, Moema, Santana do Jacaré, São Gonçalo do Pará e São José da Varginha.

Em 46 (85,1\%) dos 54 municípios, ocorreram notificações, totalizando 850 para o período de 2000 a 2003. Analisando-se todos os anos, foram realizadas 835 visitas domiciliares, representando $98,2 \%$ de cobertura às notificações. Dos atendimentos realizados pelos funcionários do PCDCh, 233 (27,9\%) confirmaram a presença de triatomíneos na UD notificante. Dentre os triatomíneos capturados pela população (notificados), 72,9\% estavam no intrado- micílio, enquanto, nos atendimentos às notificações, essa situação foi modificada, pois $69,1 \%$ dos focos foram encontrados no peridomicílio. Verifica-se que existe diferença significativa entre a forma de captura e o local de captura dos triatomíneos $(\mathrm{p}<0,05)$. Além disso, o número de insetos capturados foi maior na notificação (Tabela 1).

Dados referentes à pesquisa integral realizada em oito municípios no ano de 2003 (Córrego Fundo, Conceição do Pará, Estrela do Indaiá, Iguatama, Itaúna, Medeiros, São Francisco de Paula e Tapiraí) revelam que, das 11.265 UDs trabalhadas, 45 estavam positivas (índice de infestação domiciliar de $0,12 \%$ ).

Tanto na notificação quanto no atendimento, a espécie prevalente na região foi $P$. megistus (Tabela 2), estando presente em 43 dos 46 municípios onde houve notificações.

A proporção de triatomíneos fêmeas foi significativamente maior do que a de machos, tanto na notificação como no atendimento $(\mathrm{p}<0,05)$. Quanto às ninfas, essas foram capturadas em maior número durante o atendimento (Tabela 3).

Para a pesquisa de flagelados semelhantes ao T. cruzi, foram examinados 752 exemplares, representando $49,1 \%$ do total de insetos encontrados. Os exemplares não examinados encontravam-se mortos ou secos. Apenas 1,3\% dos examinados estavam positivos para $T$. $c r u$ $z i$, sendo todos exemplares de P. megistus.

Na Figura 2 foram analisados dados referentes ao ano de 2002, notando-se que o número de adultos, em relação ao número de ninfas, aumentou nos meses quentes do ano, principalmente a partir do mês de novembro até fevereiro.

\section{Discussão e conclusões}

Atualmente, 280 municípios estão sob vigilância em Minas Gerais 26, incluindo, dentre esses,

Distribuição dos triatomíneos segundo a atividade e local de captura em 46 municípios de Minas Gerais, Brasil, no período de 2000-2003.

\begin{tabular}{|c|c|c|c|c|c|c|}
\hline \multirow[t]{2}{*}{ Atividade } & \multicolumn{6}{|c|}{ Local de captura } \\
\hline & Intradomicílio & $\%$ & Peridomicílio & $\%$ & Total & $\%$ \\
\hline Notificação & 703 & 69,1 & 315 & 30,9 & 1.018 & 66,5 \\
\hline Atendimento & 139 & 27,1 & 374 & 72,9 & 513 & 33,5 \\
\hline Total & 842 & 55,0 & 689 & 45,0 & 1.531 & 100,0 \\
\hline
\end{tabular}


Triatomíneos coletados segundo a espécie e local de captura, em 46 municípios de Minas Gerais, Brasil, entre 2000 e 2003.

\begin{tabular}{|c|c|c|c|c|c|}
\hline Espécie & Intradomicílio & $\%$ & Peridomicílio & $\%$ & Total \\
\hline P. megistus & 787 & 54,7 & 653 & 45,3 & 1.440 \\
\hline P. diasi & 37 & 68,5 & 17 & 31,5 & 54 \\
\hline R. neglectus & 13 & 65,0 & 7 & 35,0 & 20 \\
\hline T. sordida & 5 & 29,4 & 12 & 70,6 & 17 \\
\hline Total & 842 & - & 689 & - & 1.531 \\
\hline
\end{tabular}

Tabela 3

Proporção de triatomíneos, em 54 municípios de Minas Gerais, Brasil, de acordo com o estádio evolutivo e a forma de captura, entre os anos de 2000 e 2003.

\begin{tabular}{|c|c|c|c|c|c|c|}
\hline \multirow[t]{2}{*}{ Estádio evolutivo } & \multicolumn{4}{|c|}{ Forma de captura } & \multirow[t]{2}{*}{ Total } & \multirow[t]{2}{*}{$\%$} \\
\hline & Notificação & $\%$ & Atendimento & $\%$ & & \\
\hline Machos & 343 & $82,5(34,0)$ & 71 & $17,5(13,6)$ & 414 & $100,0(27,4)$ \\
\hline Fêmeas & 512 & $78,1(50,7)$ & 140 & $21,8(26,9)$ & 652 & $100,0(45,6)$ \\
\hline Ninfas & 155 & $33,3(15,3)$ & 310 & $66,7(59,5)$ & 465 & $100,0(30,4)$ \\
\hline Total geral & 1.010 & $66,0(100,0)$ & 521 & $44,0(100,0)$ & 1.531 & $100,0(100,0)$ \\
\hline
\end{tabular}

os 54 municípios da DADS de Divinópolis. Os serviços realizados pela DADS em questão têm como prioridade o atendimento às notificações realizadas pela população e também a busca ativa, através de PI dos municípios.

O presente trabalho faz parte de um estudo prospectivo de funcionamento do PCDCh em uma região com vigilância instalada e apresentou dificuldades metodológicas comuns em estudos que se valem de dados secundários. Mas, mesmo assim, justifica-se como um primeiro passo de avaliação operativa da vigilância e análise da tendência de domiciliação das espécies na região.

A espécie mais detectada no âmbito domiciliar, por moradores e agentes de vigilância na área estudada, foi $P$. megistus. Essa espécie habita matas úmidas, ocorrendo em todos os tipos de florestas existentes no Brasil extra-amazônico. Dos elementos climáticos, somente a aridez exerce ação limitante sobre a dispersão desse triatomíneo 27,28. No Estado de Minas Gerais, o inseto tem sido freqüentemente capturado em domicílios e também em biótopos naturais 29,30. Eliminado o T. infestans de Minas Gerais, $P$. megistus voltou a constituir-se no vetor mais importante da doença de Chagas hu- mana na zona oeste do Estado, sendo a espécie mais capturada no âmbito domiciliar 31,32.

$\mathrm{O}$ encontro de ninfas de $P$. megistus em $32,3 \%$ das capturas evidencia o processo de domiciliação dessa espécie na região. Verificou-se que a infestação por $P$. megistus ocorreu principalmente no peridomicílio, um ambiente problemático para o controle químico, pois, nesse local, o efeito residual dos inseticidas é menor 33. As presentes observações reforçam trabalhos anteriores, indicando que os processos de infestação e colonização por P.megistus na região se originam de focos silvestres, principalmente, por meio de fêmeas já fecundadas que migram através de vôo para as UDs, instalando-se preferencialmente no peridomicílio, de onde podem invadir a habitação, principalmente a partir do mês de outubro até o mês de fevereiro 29,34.

Outras espécies encontradas na região foram $P$. diasi e $R$. neglectus. Dessas espécies, todos os insetos capturados eram adultos, e nenhum estava positivo para T. cruzi, o que sugere sua origem silvestre, baixo poder de colonização e associação básica com aves ou outras fontes não infectadas pelo parasito 28,35 . Vale salientar que esses triatomíneos foram notificados em 35 municípios nos últimos quatro 
Proporção mensal de ninfas e adultos de P. megistus capturados durante os anos 2002 e 2003 nos 54 municípios coordenados pela DADS Divinópolis. Minas Gerais, Brasil.

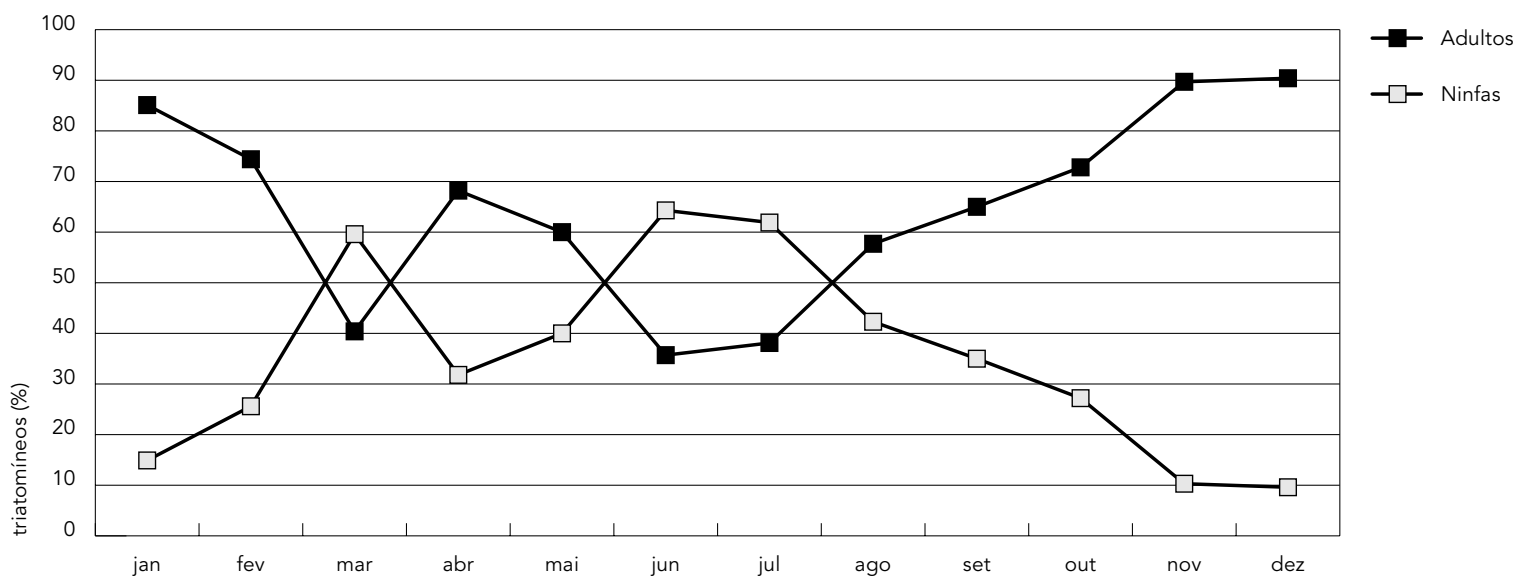

anos, o que mostra a sua grande dispersão na região. Já $T$. sordida mostrou-se focalizado e menos presente na área estudada, em contraste com outras regiões de estudo, como o norte e Triângulo Mineiro. O fato de T. sordida invadir esporadicamente ecótopos artificiais pode significar, em algum momento, um risco de colonização, devendo ser objeto de continuada monitorização 10. Quanto ao encontro do maior número de ninfas de $P$. megistus durante o atendimento, isso sugere que a população está mais sensibilizada (ou preparada) para a captura de insetos adultos, tanto pela maior facilidade de encontro desses devido ao seu tamanho, como também pelo maior conhecimento desse estádio evolutivo. Esse fato, que se repete em outras áreas como São Paulo, é importante para o aprimoramento da vigilância, em termos das atividades educativas, devendo ser levado em conta nos trabalhos e materiais produzidos e nas informações repassados às comunidades-alvo 36.

Em oito municípios, não foram realizadas notificações, o que pode explicar-se por: (a) a ausência (ou mui diminuta ocorrência) de insetos invadindo domicílios nessas localidades; (b) pouco conhecimento e estímulo da população sobre a captura e denúncia dos vetores da doença de Chagas e (c) desativação do PCDCh municipal em termos estruturais. Todas essas possibilidades são prováveis, visto que Araújos (Figura 1), por exemplo, faz divisa com cinco municípios que tiveram notificações regulares no período estudado, sendo esse o único município sem nenhuma notificação. Em sua formulação, o presente trabalho prevê análise específica de cada um desses municípios, através de supervisão direta e de reuniões com os responsáveis locais pela vigilância e controle, além de instalação de pesquisa direta (busca ativa) de triatomíneos nos mesmos, utilizando-se equipes regionais e municipais, conforme as normativas do Ministério da Saúde 37.

É relevante notar que o número de atendimentos cobriu 83,3\% das notificações. Esse dado é importante, pois, para a população colaborar com a fase da vigilância, é necessária tanto a materialização de uma resposta específica ao ato de notificação, quanto que os atendimentos sejam realizados o mais precocemente possível 38. A confirmação de focos ocorreu em $27,9 \%$ das notificações, o que sugere que os focos geralmente têm pequena densidade, e que os adultos capturados pela população correspondem a insetos isolados e recém migrados de focos silvestres 39,40. Outro fator relevante é que o encontro de triatomíneos durante o atendimento à população é muito mais numeroso se comparado às atividades de pesquisa integral. Com efeito, no ano de 2002, considerando oito municípios, a detecção de triatomíneos só ocorreu em $0,12 \%$ das casas pesquisadas.

Pelos dados apresentados pode-se inferir que: T. infestans foi efetivamente eliminado da 
área estudada na última década, não sendo detectado nenhum exemplar da espécie em inúmeras e seguidas pesquisas ou notificações realizadas ao longo da última década, o que foi reiterado na presente análise 37 .

Além disso, o presente trabalho indica claramente a manutenção de espécies nativas como as espécies T. sordida, P. diasi e R. neglectus, o que coincide com informações prévias e mostra a residualidade e a ampla dispersão dessas espécies na região, assim como seu pequeno potencial invasivo para ecótopos artificiais 16,28. Confrontando com dados históricos, especialmente aqueles mais detalhados do Município de Bambuí, vê-se que essa situação permanece a mesma há, pelo menos, duas ou três décadas, o que reforça a idéia de que, na região, tais espécies (incluindo outras de ainda menor expressão epidemiológica relativamente à doença humana, como T. pseudomaculata, Psammolestes tertius e Cavernicola sp.) muito dificilmente virão a constituir-se em perigo de transmissão do T. cruzi ao homem na área, mormente se mantida uma vigilância entomológica efetiva e continuada 12,14,41,42. Já $P$. megistus continua prevalecendo na região estudada e, embora seja detectado em baixas densidades, ainda foi detectado em 46 dos 54 municípios. O nível de infecção para flagelados semelhantes ao T. cruzi dos triatomíneos capturados foi semelhante $(1,3 \%)$ quando comparamos com outros autores 43 , isso pode estar significando tanto um progressivo esvaziamento do ciclo doméstico do parasito, como a redução de mamíferos infectados em ecótopos naturais de origem dos triatomíneos capturados 4,40. Como esperado e também observado em outras regiões, o principal âmbito da detecção de triatomíneos pela população segue sendo o intradomiciliar, também predominando a captura de insetos adultos 32,36,40. Como na melhor expectativa se deseja que as atividades de vigilância a longo prazo se sustentem e baseiem-se fundamentalmente na participação comunitária, esse ponto se reveste de especial importância, particularmente no que toca a atividades de informação e educação sanitária (ensinar a reconhecer ninfas, voltar mais atenção ao peridomicílio etc.) 9,32. De modo geral, os dados aferi- dos estão a indicar que os focos domiciliares de P. megistus, nessa região, atualmente, sejam adventícios, originados de migração ativa a partir de focos silvestres justa-domiciliares, especialmente por meio de fêmeas previamente fecundadas que saem a migrar mais freqüentemente no período de outubro a março, dado que pode ser confirmado na Figura 2, quando predominam essas formas evolutivas, sugerindo também que a espécie apresenta uma única geração anual. A Figura 2 também mostra coerência com os dados já observados na região, sendo que as notificações e as capturas de adultos predominam nos meses úmidos do ano e assim concordam com dados laboratoriais e buscas ativas no Município de Bambuí 39,40,44.

Foram capturados triatomíneos em grande parte dos municípios incluídos no estudo, e a participação da população mostrou-se importante, sendo essa a responsável pela maioria dos insetos capturados. O encontro, principalmente de $P$. megistus, invadindo e colonizando o ambiente domiciliar, reforça a importância da manutenção da vigilância da endemia chagásica nos sistemas locais de saúde nessa e em regiões semelhantes, com a participação da comunidade e também dos agentes municipais devidamente capacitados. A esses cabe sensibilizar a população e realizar os atendimentos à mesma quando solicitados, o que envolve atividades de capacitação e supervisão periódicas, hoje, pertinentes aos estados. Daí serem necessárias políticas municipais e estaduais de apoio e sustentação ao PCDCh, no contexto da descentralização das ações de saúde, tendo em vista a desativação do controle de endemias em nível federal pela FUNASA 4,12,23. Acresce que esse programa vem sendo negligenciado nos últimos tempos, devido aos baixos níveis de transmissão e também por questões político-administrativas, fatos esses que demandam atenção das autoridades, continuada monitorização e apoio direto aos municípios ${ }^{2}$. Assim, o maior desafio para o controle da transmissão vetorial da doença será a continuidade da vigilância, já que as invasões domiciliares, dadas por insetos infectados, provenientes de ambiente silvestre, sempre será uma possibilidade. 


\section{Resumo}

Em Minas Gerais, Brasil, após a eliminação do Triatoma infestans, realizada pelo Programa de Controle da Doença de Chagas (PCDCh), algumas espécies, como Panstrongylus megistus, merecem maior atenção da vigilância entomológica. Com a reforma sanitária, as ações do PCDCh couberam aos municípios, e alguns têm encontrado dificuldades administrativas e operacionais para a realização e/ou manutenção do programa. O presente trabalho objetivou analisar a vigilância entomológica da doença de Chagas nos 54 municípios supervisionados pela Diretoria de Ações Descentralizadas da Saúde de Divinópolis, entre 2000 e 2003. Em 46 municípios, foram capturados 1.531 triatomíneos nesse período, sendo que $94,1 \%$ dos insetos eram exemplares de $\mathrm{P}$. megistus. O índice de infecção para $\mathrm{T}$. cruzi foi de 1,3\% nos insetos examinados. Ao todo, foram realizadas 850 notificações distribuídas em 46 municipios, que resultaram em 835 atendimentos, $o$ que mostra que o PCDCh está ativo e eficiente na maioria dos municípios analisados. Constatou-se que os triatomíneos continuam a invadir e eventualmente colonizar o ambiente domiciliar, ainda que em baixa densidade, demonstrando a importância da manutenção e do aprimoramento da vigilância entomológica na região.

Doença de Chagas; Vigilância Epidemiológica; Panstrongylus

\section{Colaboradores}

M. M. Villela e J. C. P. Dias realizaram a elaboração do artigo. J. M. B. Souza, V. P. Mello e B. V. M. Azeredo supervisionaram as equipes de campo da DADS de Divinópolis, por meio de visitas, palestras e reuniões com os agentes do PCDCh e secretários da saúde de todos os municípios. Além disso, a confecção dos relatórios e resumos, que serviram de base para a redação deste artigo, foi realizada por ambos.

\section{Agradecimentos}

Este trabalho é parcialmente subsidiado pelo Programa Especial de Pesquisa e Treinamento em Doenças Tropicais - TDR - (Organização Mundial da Saúde/ Banco Mundial/Programa das Nações Unidas para o Desenvolvimento).

\section{Referências}

1. World Health Organization. Control of Chagas disease. Geneva: World Health Organization; 2002. (WHO Technical Report Series 811).

2. Dias JCP. Doença de Chagas. Ambiente, participação e Estado. Cad Saúde Pública 2001; 17 Suppl: 165-9.

3. Silveira AC, Rezende DF. Epidemiologia e transmissão vetorial da doença de Chagas no Brasil. Rev Soc Bras Med Trop 1994; 27 Suppl 3:11-22.

4. Dias JCP, Coura, JR. Epidemiologia. In: Dias JCP, Coura JR, organizadores. Clínica e terapêutica da doença de Chagas. Rio de Janeiro: Editora Fiocruz; 1997. p. 33-65.

5. Martins AV, Versiani V, Tupinanbá A. Estudo sobre a tripanossomíase americana em Minas Gerais, Brasil. Mem Inst Oswaldo Cruz 1946; 35:285.

6. Silveira AC, Feitosa VR, Borges R. Distribuição de triatomíneos capturados no ambiente domiciliar no período de 1975/1983, no Brasil. Rev Bras Malariol Doenças Trop 1984; 36:15-312.

7. Chagas C. Nova espécie mórbida do homem produzida por um trypanosoma (Trypanossoma cruzi). Nota prévia. Brasil Médico 1909; 230:161.

8. Filho JC, Silveira AC. Distribuição da doença de Chagas no Brasil. Revista da Sociedade Brasileira de Malariologia e Doenças Tropicais 1979; 31:8698.

9. Dias JCP. Control of Chagas disease in Brazil. Parasitol Today 1987; 3:336-41.

10. Dias JCP. O controle da doença de Chagas no Brasil. In: Silveira AC, Arias AR, Segura E, Guilén G, Russomando G, Schenone H, et al., organizadores. O controle da doença de Chagas nos países do Cone Sul da América: história de uma iniciativa internacional 1991/2001. Uberaba: Faculdade de Medicina do Triângulo Mineiro; 2002. p. 145-237.

11. Lucena DT. Ecologia dos triatomíneos do Brasil. Revista da Sociedade Brasileira de Malariologia e Doenças Tropicais 1959; 11:617-8.

12. Secretaria do Estado da Saúde de Minas Gerais/ Fundação Nacional da Saúde/Fundação Oswaldo Cruz. Interrupção da transmissão vetorial da doença de Chagas por Triatoma infestans em Minas Gerais. Relatório técnico da comissão de avaliação e certificação de eliminação da transmissão vetorial da doença de Chagas em Minas Gerais. Belo Horizonte: Secretaria do Estado da Saúde de Minas Gerais/Fundação Nacional da Saúde/Fundação Oswaldo Cruz; 2001.

13. Dias E. Um ensaio profilático de moléstia de Chagas. Rio de Janeiro: Imprensa Nacional; 1945.

14. Dias E. Profilaxia da doença de Chagas. Resumo das principais atividades do Centro de Estudos do Instituto Oswaldo Cruz em Bambuí, MG. Brasil Médico 1946; 60:161-3.

15. Dias E. Considerações sobre a importância da doença de Chagas em Minas Gerais e estados vizinhos: necessidade urgente de ser desenvolvido um estudo desta endemia e serem tomadas medidas para combatê-la. Brasil Médico 1949; 63: 200-17.

16. Dias JCP. Doença de Chagas em Bambuí, Minas Gerais, Brasil. Estudo clínico-epidemiológico a 
partir da fase aguda entre 1940 e 1982 [Tese de Doutorado]. Belo Horizonte: Universidade Federal de Minas Gerais; 1982.

17. Dias JCP. Cinqüenta anos de Bambuí. Rev Soc Bras Med Trop 1993; 26 Suppl 2:4-8.

18. Dias E. Plano de erradicação dos triatomíneos domiciliares no Município de Bambuí, Minas Gerias. Jornal do Comércio 1956; 10 ago.

19. Dias E. Erradicação do Triatoma infestans. In: Proceedings of the Sixth International Congress on Tropical Medicine and Malaria. v. 3. Lisboa: s.e.; 1958. p. 200-58.

20. Dias E, Pellegrino J. Alguns ensaios com o gammexane no combate aos transmissores da doença de Chagas. Brasil Médico 1948; 62:185-91.

21. Marsden PD. The control of Latin American trypanosomiasis. Rev Soc Bras Med Trop 1997; 30: 521-7.

22. Silva EOR, Wanderley DMV, Rodrigues VLCC. Triatoma infestans: controle e eliminação da espécie no Estado de São Paulo, Brasil. Rev Soc Bras Med Trop 1998; 31:73-88.

23. Vinhaes MC, Dias JCP. Doença de Chagas no Brasil. Cad Saúde Pública 2000; 16 Suppl 2:7-12.

24. Silva RA, Bonifácio PR, Wanderley DMV. Doença de Chagas no Estado de São Paulo: Comparação entre pesquisa ativa de triatomíneos em domicílios e notificação de sua presença pela população em área sob vigilância entomológica. Rev Soc Bras Med Trop 1999; 32:653-9.

25. Ministério da Saúde. Manual de normas técnicas da campanha de controle da doença de Chagas. Brasília: Superintendência de Campanhas de Saúde Pública; 1980.

26. Moreno EC, Baracho L. Vigilância epidemiológica no Programa de Controle da Doença de Chagas em Minas Gerais, Brasil (1984-1998). Cad Saúde Pública 2000; 16 Suppl 2:113-6.

27. Forattini OP. Biogeografia, origem e distribuição da domiciliação de triatomíneos no Brasil. Rev Saúde Pública 1980; 14:265-99.

28. Carcavallo RU, Rodriguez MEF, Salvatella R, Casas SIC, Sherlock IS, Galvão C, et al. Hábitos e fauna relacionada. In: Carcavallo RU, Girón GI, Juberg J, Lent $\mathrm{H}$, organizadores. Atlas dos vetores da doença de Chagas nas Américas. Rio de Janeiro: Editora Fiocruz; 1999. p. 561-600.

29. Barretto MP, Siqueira AF, Freitas JP. Estudos sobre reservatórios e vetores silvestres do Trypanossoma cruzi. II - Encontro de Panstrongylus megistus em ecótopos silvestres no Estado de São Paulo (Hemiptera, Reduviidae). Rev Inst Med Trop São Paulo 1964; 6:56-63.

30. Freitas JLP, Siqueira AF, Ferreira OA. Investigações epidemiológicas sobre triatomíneos de hábitos domésticos e silvestres com auxílio da reação de precipitina. Rev Inst Med Trop São Paulo 1960; 2:90-9.

31. Aragão MB. Aspectos climáticos da doença de Chagas. II - Área de ocorrência do Panstrongylus megistus (Burmeister, 1835). Revista Brasileira de Malariologia 1961; 13:171-93.
32. Dias JCP, Garcia ALR. Vigilancia epidemiológica con participación comunitaria. Un programa de enfermedad de Chagas. Bol Oficina Sanit Panam 1978; 84:533-44.

33. Oliveira Filho AM. New alternatives for the control of triatomines in peridomestic buildings. Rev Soc Bras Med Trop 1989; 22:53-7.

34. Fernandes AJ, Diotaiuti L, Dias JCP, Romanha AJ, Chiari E. Inter-relações entre os ciclos de transmissão do Trypanossoma cruzi no Município de Bambuí, Minas Gerais, Brasil. Cad Saúde Pública 1994; 10:473-80.

35. Carcavallo RU, Rocha DS, Girón I, Sherlock IA, Galvão C, Martinez A, et al. Fontes e padrões alimentares. In: Carcavallo RU, Girón GI, Juberg J, Lent $\mathrm{H}$, organizadores. Atlas dos vetores da doença de Chagas nas Américas. Rio de Janeiro: Editora Fiocruz; 1999. p. 537-60.

36. Dias JCP. The impact of Chagas disease control in Latin America: a review. Mem Inst Oswaldo Cruz 2002; 97:603-12.

37. Ministério da Saúde. Proposta para a certificação da interrupção da transmissão vetorial da doença de Chagas por Triatoma infestans no Brasil. Brasília: Fundação Nacional de Saúde; 2000.

38. Dias JCP. Chagas disease control in Brazil: which strategy after the attack phase? Ann Soc Belg Med Trop 1991; 71:75-86.

39. Dias JCP. Vigilância epidemiológica da doença de Chagas. Cad Saúde Pública 2000; 16 Suppl 2:4359.

40. Wanderley DMV. Perspectivas de controle da doença de Chagas no Estado de São Paulo [Tese de Doutorado]. São Paulo: Faculdade de Saúde Pública, Universidade de São Paulo; 1994

41. Dias E. O Centro de Estudos e Profilaxia da Doença de Chagas em Bambuí, Estado de Minas Gerais. (Notícia histórica em homenagem ao Prof. Henrique Aragão). Mem Inst Oswaldo Cruz 1956; 54:309-57.

42. Dias E, Pinto OS. Combate aos triatomas com BHC na cidade de Bambuí, Minas Gerais. Rev Bras Malariol Doenças Trop 1952; 4:22-46.

43. Costa J, Almeida CE, Dotson EM, Lins A, Vinhaes M, Silveira AC. The epidemiologic importance of Triatoma brasiliensis as a Chagas disease vector in Brazil: a revision of domiciliary captures during 1993-1999. Mem Inst Oswaldo Cruz 2003; 98 : 443-9.

44. Forattini OP. Aspectos ecológicos da tripanossomíase americana. XI - Domiciliação de Panstrongylus megistus e potencial enzoótico. Rev Saúde Pública 1977; 11:527-50.

Recebido em 15/Set/2004

Versão final reapresentada em 22/Dez/2004

Aprovado em 04/Jan/2005 